\title{
Interfaces
}

\section{'Many dark ribbons' ou le format mis en fiction : The Golden Apples de Eudora Welty}

Jean-Marc Victor

\section{(2) OpenEdition}

1 Journals

Édition électronique

URL : https://journals.openedition.org/interfaces/3249

DOI : 10.4000/interfaces.3249

ISSN : 2647-6754

Éditeur :

Université de Bourgogne, Université de Paris, College of the Holy Cross

\section{Référence électronique}

Jean-Marc Victor, «'Many dark ribbons' ou le format mis en fiction: The Golden Apples de Eudora Welty », Interfaces [En ligne], 46 | 2021, mis en ligne le 15 décembre 2021, consulté le 18 juin 2022 URL : http://journals.openedition.org/interfaces/3249; DOI : https://doi.org/10.4000/interfaces.3249

Ce document a été généré automatiquement le 18 juin 2022

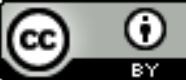

Les contenus de la revue Interfaces sont mis à disposition selon les termes de la Licence Creative Commons Attribution 4.0 International. 


\section{'Many dark ribbons' ou le format mis en fiction: The Golden Apples de Eudora Welty}

Jean-Marc Victor

1 Face à la tyrannie éditoriale du roman dans le champ de la fiction américaine, un certain nombre d'auteurs de la première moitié du $\mathrm{XX}^{\mathrm{e}}$ siècle s'écartent des conventions formelles qui président aux modalités du récit en explorant les possibilités offertes par le «cycle de nouvelles" (short story cycle). On songe notamment à Sherwood Anderson et son célèbre Winesburg, Ohio paru en 1919, bientôt suivi de In Our Time de Hemingway en 1925, The Pastures of Heaven de Steinbeck en 1932, ou encore The Unvanquished de Faulkner en 1938, pour n'en citer que quelques-uns parmi les plus connus de l'ère moderniste ${ }^{1}$.

Dans le cadre de ce format narratif singulier, chaque nouvelle du cycle peut se lire de manière autonome, mais son sens se trouve simultanément modifié et/ou accru par les rapports qu'elle entretient avec les autres textes du volume ${ }^{2}$. La lecture se fait sur un mode discontinu et fragmentaire: l'unité n'étant pas donnée au départ, elle est à reconstruire (et souvent partiellement, d'ailleurs) au gré de divers types d'échos dont l'identification sollicite, beaucoup plus fortement que dans le cas du roman, la participation active du lecteur, lequel ne sait pas d'emblée à quel type d'objet littéraire il se trouve confronté.

3 On se propose d'analyser ici la complexité des jeux de format induits par la lecture d'un cycle de nouvelles exemplaire à plus d'un titre: The Golden Apples de Eudora Welty (1949). Dans cette chronique de Morgana, petite ville imaginaire du Mississippi, saisie à travers le prisme de sept « moments » vécus par des protagonistes à la fois différents et récurrents d'une nouvelle à l'autre, Welty signe un récit épisodique couvrant une quarantaine d'années, depuis le début du XXe siècle jusqu'à la fin des années 1940. On y suit, selon un mode intermittent, la vie de quelques personnages, pour la plupart issus des principales familles de Morgana, lesquels apparaissent, disparaissent et reviennent au gré d'une construction où alternent temps forts et ellipses. Welty tourne ici 
délibérément le dos au format romanesque. En mars 1949, peu avant la parution de l'ouvrage, celle qui s'est alors davantage illustrée par sa prédilection pour le format court $^{3}$ va jusqu'à mettre en garde son agent contre toute publicité qui, à des fins commerciales, décrirait The Golden Apples comme roman :

I'l turn it down if that's the catch and maybe they'll turn back the book but nothing changes the book into a novel or play or poem, if it's a book of related stories, then it is. (Maybe we could call it something like 'Variations on a Portfolio' or something from the other arts where such groupings are more common? (Kreyling 146)

De variation, il est d'ailleurs largement question à l'intérieur même du cycle, où l'instabilité du format est frappante: la longueur des textes constitutifs de l'ouvrage varie de 10 à 56 pages, chaque nouvelle est elle-même subdivisée en parties (de deux à sept, ce qui accentue considérablement l'impression de discontinuité à la lecture), et l'unité de lieu, communément attendue d'une telle chronique, est brutalement rompue dans l'avant-dernière nouvelle, dont l'action se déroule non plus dans le Mississippi mais à San Francisco.

On se demandera donc comment ce format cyclique instable interagit avec la ligne thématique du livre, tendue entre liaison et déliaison et, du même coup, en quoi il peut mener à une réflexion plus large sur les modalités de réception de cet objet narratif en rupture avec les normes romanesques, en induisant par exemple certaines pratiques de lecture (et même de relecture) qui vouent le lecteur à une expérience alternant reconnaissance et dépaysement, convergence et diffraction. On tentera ainsi d'examiner les enjeux esthétiques de ce format singulier, le plus central étant de thématiser son propre mode de fonctionnement au sein d'un récit fictionnel constamment préoccupé de jonction et de disjonction, reflétant ainsi dans les moindres replis de sa structure formelle et de sa teneur diégétique les enjeux inhérents à l'acte de lecture, entre liberté et contrainte.

\section{De Winesburg, Ohio à Morgana, Mississippi}

Le genre du cycle de nouvelles est particulièrement prospère aux Etats-Unis à partir de la fin du XIX siècle. C'est donc contre toute réalité historique que Sherwood Anderson se targue d'en avoir posé les bases en 1919 :

What is wanted is a new looseness, and in Winesburg I had made my own form. There were individual tales but all about lives in some way connected. By this method I did succeed, I think, in giving the feeling of the life of a boy growing to manhood in a town. (Anderson 1942, 289)

7 S'il cerne avec justesse les deux termes qui polarisent le genre («looseness» et "connection»), il ne fait pourtant qu'emboîter le pas à des prédécesseurs moins illustres, en particulier des femmes telles que Sara Orne Jewett, qui publiait dès 1896 son cycle de nouvelles The Country of the Pointed Firs ${ }^{4}$. Il reste que la méthode qu'il s'assigne dans Winesburg pourrait aussi bien rendre compte de l'entreprise de Welty trente ans plus tard, et plus généralement des nombreux cycles évoquant la vie d'une petite ville américaine : "taking a related group of people, their lives touching, never quite touching ", voilà le projet tel qu'il le décrit lui-même (Mann 11). Ces vies qui se touchent sans se toucher suggèrent à la fois contiguïté et effleurement, deux principes à l'œuvre non seulement dans la diégèse, mais dans la structuration formelle du cycle et dans les modalités de lecture qu'il suscite. 
8 Sherwood Anderson, s'il ne fut pas l'inventeur du genre aux Etats-Unis, contrairement à ce qu'il prétend, eut toutefois à son propos une intuition géniale : « Do we not live in a great loose land of many states, and yet all these states together do make something, a land, a country. I submit that the form of my Winesburg tales (...) may offer a suggestion to other writers ", écrit-il encore (Schevill 96). La forme du cycle de nouvelles serait donc une sorte de reflet particulièrement adapté à la représentation de la réalité de la nation américaine dans son fédéralisme même, chaque texte obéissant à la fois individuellement à la logique de lois qui lui sont propres, mais aussi collectivement à celle d'enjeux d'un niveau supérieur fondant l'unité de l'ensemble. Il faut attendre 1995 pour lire sous la plume de Gerald Kennedy la concrétisation théorique de l'intuition précoce d'Anderson :

the proliferation of short story sequences 5 is truly a global phenomenon, still the pragmatic affinity for short stories that shaped the literature of the United States in the nineteenth century seems to persist in our national avidity for organized story collections. Perhaps the very determination to build a unified republic out of diverse states, regions, and population groups - to achieve the unity expressed by the motto e pluribus unum - helps to account for this continuing passion for sequences. (Kennedy viii)

9 Faire un à partir d'une pluralité, ambition démocratique de la nation américaine, voilà donc un projet que la littérature américaine s'assignerait indirectement à son tour sous la forme de cette communauté de textes que constitue le cycle de nouvelles.

La fine connaissance qu'avait Welty du Winesburg d'Anderson est un fait avéré (Welty, Occasions 196) ${ }^{6}$. Pour autant, ce n'est pas consciemment qu'elle reprend ce format à son compte. Elle explique en effet dans son autobiographie que les liens entre les histoires constitutives de The Golden Apples ne lui sont apparus qu'à mi-parcours dans la rédaction de l'ensemble, alors même qu'elle avait commencé à les publier séparément dans des revues (comme Anderson l'avait fait avant elle avec ses « Winesburg tales ») ${ }^{7}$ sans avoir conscience, dit-elle, qu'elle composait là les fragments d'un tout plus vaste dont la cohérence ne s'imposerait à elle que dans un second temps: "They touched on every side ", écrit-elle, reprenant ainsi les motifs d'effleurement et de contiguïté déjà évoqués par Anderson. «In writing, as in life, poursuit Welty, the connections of all sorts of relationships and kinds lie in wait of discovery " (Beginnings 108).

Il est remarquable que Welty fasse l'expérience de ces liens souterrains entre les textes presque malgré elle, ou plus exactement en se plaçant nécessairement dans la position de lectrice (voire de relectrice) de son propre matériau narratif. L'implicite de cette découverte est bien sûr qu'un cheminement similaire est attendu du lecteur à son tour : à nous de faire de même, de jouer le jeu selon les mêmes termes, de revenir sur notre lecture pour laisser affleurer des points de contact qui n'étaient pas visibles au départ ${ }^{8}$. Dans leur singularité et dans leur pluralité, les textes du cycle nous mettent au défi, ils nous attendent (" in wait of discovery»), ils nous font place en tant que partenaire privilégié dans la création partiellement rétrospective d'un certain degré d'unité thématique et formelle. La participation du lecteur est donc programmatique dans la genèse $\mathrm{du}$ format cyclique, comme le confirment, au-delà de la querelle terminologique, toutes les entreprises concurrentes de définition du cycle de nouvelles (Ingram 19; Luscher 148-49; Mann 18 ; Kennedy ix), lesquelles s'accordent au moins à placer le lecteur au cœur de l'esthétique de ce format littéraire mêlant l'un et le multiple, Kennedy étant sans doute celui qui résume le mieux cette intuition : «One must concede at last that textual unity [within the cycle], like beauty, lies mainly in the eye of the beholding reader $"$ (Kennedy ix). 
12 Ainsi, le cycle apparaît d'emblée comme un format narratif qui rend le lecteur excessivement conscient de sa propre activité de lecture, le conduisant presque à chaque page à se demander ce que c'est vraiment que de lire. Or, étymologiquement, lire est affaire d'assemblage : comme le rappelle Littré, c'est « connaître les lettres et savoir les assembler en mots ». L'origine latine du verbe renvoie à legere: au sens propre, recueillir, amasser en cueillant, rassembler; d'où le sens dérivé de "recueillir par les yeux » (Gaffiot), c'est-à-dire lire. Lire The Golden Apples, c'est donc faire cueillette dans un sens presque littéral, mais c'est aussi, plus indirectement, s'interroger sur la manière d'assembler les fruits de cette lecture - ou pour le dire autrement, examiner à tout moment chaque pomme et sa place dans le panier.

Certes, la saveur sudiste de ces fruits est indéniable : il s'agit d'interroger les modes de vie et de relation au sein d'une petite ville du Mississippi, comme l'avait fait Anderson dans le Midwest, ou Jewett en Nouvelle-Angleterre ${ }^{9}$. Mais au fond, la question récurrente du rapport entre l'individu et la communauté se charge immédiatement d'une valeur métafictionnelle puisque l'habitant se confronte à l'emprise de sa communauté comme la nouvelle se confronte à son appartenance au cycle. Il s'agit donc de se demander comment Welty, dans la matière même de l'histoire d'un groupe humain, s'approprie le format cyclique qui lui préexiste et en quoi il lui fournit matière à une sorte de métafiction dont la lecture est l'enjeu. Comme nous y invite la double dimension de chaque texte, autonome et interdépendant, il convient à présent de déceler dans chacune des nouvelles de The Golden Apples les traces d'une mise en fiction des modalités génériques $d u$ cycle, tout en se penchant sur quelques motifs transversaux qui, de texte en texte, donnent métaphoriquement et narrativement forme à une théorie de la lecture.

\section{«Shower of Gold » ou l'épreuve du double}

«Shower of Gold », nouvelle inaugurale, est d'emblée placée sous le signe de la couture. Le jour d'Halloween où King MacLain, époux volage de Miss Snowdie et incorrigible fugueur, choisit de rendre visite à l'épouse qu'il a abandonnée enceinte, et qui a donné naissance à deux jumeaux qu'il n'a jamais rencontrés, elle reçoit sa voisine Miss Katie Rainey, narratrice de la nouvelle, pour faire avec elle des travaux d'aiguille. Ironie du sort, les deux femmes n'ont même pas le temps d'apercevoir le visiteur que les intrépides jumeaux masqués le mettent déjà en fuite. L'événement est si subreptice que Morgana en vient à douter de sa réalité. Le même jour, sur ces entrefaites, Virgie, fille de Miss Katie, avale un bouton qu'il faut lui faire régurgiter pour éviter qu'elle ne s'étouffe. Parviendra-t-on à recoudre les éléments épars de ce récit avorté et hétéroclite ? Tel est le défi lancé au lecteur dans cette nouvelle. Comment réconcilier le fait narratif et sa mise en doute, la scélératesse objective de King et son statut pourtant légendaire dans la communauté, qui en fait volontiers le géniteur d'une flopée d'enfants disséminés dans tout le comté ? A l'évidence, Welty invite par anticipation son lecteur à une lecture-couture, un ravaudage, une reprise, et le mot s'entend dans son sens textile comme dans son acception littérale.

De plus, tout dans cette nouvelle est double : jumeaux, masques, structure bipartite, réalité avérée se frottant à un irréel légendaire ou fantasmé. Les personnages aussi sont doubles. Ainsi, le jour où elle vient annoncer à sa voisine qu'elle est enceinte, Snowdie, bien ancrée dans son Mississippi natal, est en même temps une réincarnation de Danaé 
rendue fertile par une pluie d'or: "It was like a shower of something had struck her, like she'd been caught out in something bright » $(1949,266)$. L'événement fait du même coup de King, séducteur invétéré de la chronique locale, un avatar de Zeus. Chacun des jumeaux est lui-même, mais aussi le masque qu'il porte et le double de l'autre et encore la réplique exacte du père: "they were both King all over again" $(1949,267)$. Et ce principe de réduplication s'emballe. Double aussi, Morgana est à la fois une bourgade typique du Sud, qui tient son nom de l'illustre lignée locale des Morgan, et un mirage à la surface de l'eau, comme l'évoque l'expression "Fata Morgana", qui décrit une illusion d'optique. L'omniprésence du double signale les deux pans d'un vêtement narratif mal ajointé qui laisserait toujours bâiller quelque chose entre un ici et sa transfiguration, entre le local et l'universel, entre l'unique et le commun, ce qui fait aussi écho à la nature double de chaque nouvelle dans le cycle, évoquée ici par Lundén :

the stories of the composite exist simultaneously as self-contained entities and as interconnected parts of a larger whole. The reader experiences a 'doubleness' present in the individual stories. Ultimately the most characteristic feature of the short story composite is this 'double' existence of the stories, and the tension that emerges from that doubleness. (46)

Le bouton avalé par Virgie désigne, quant à lui, l'attache manquante, l'impossible fermeture de la forme, l'irréductible bâillement de l'habillage narratif, à moins qu'on ne tienne Virgie par les pieds pour qu'elle restitue le lien qui fait défaut comme on revient sans cesse sur le texte dans une relecture-régurgitation en quête de signes qui aideraient à la suture de ce format toujours béant.

\section{« June Recital » ou la maison aux sept... fenêtres}

17 "June Recital », la plus longue des sept nouvelles, et de loin la plus complexe dans l'intrication de son réseau signifiant, repose sur une architecture à l'image de la maison qui lui sert de cadre, miroir métatextuel reflétant l'architecture même du cycle. Deux enfants de nouveau, cette fois Loch et Cassie Morrison, observent les étranges agissements de personnages énigmatiques qui vont et viennent dans la maison voisine, celle des MacLain devenue vide des années après "Shower of Gold». Nous sommes au cœur d'un maillage étroit de la topographie urbaine placée sous le signe de la contiguïté, comme le recueil dans son ensemble : tout se touche et s'effleure, et la maison MacLain côtoie la maison Morrison comme «Shower of Gold» voisine avec « June Recital ».

18 Muni d'un télescope, Loch épie l'ancienne locataire des lieux, Miss Eckhart, qui fut professeur de piano à Morgana, au moment où elle revient mettre le feu à la demeure, celle-là même où Cassie suivait autrefois ses leçons de musique. Tandis que la vieille femme devenue folle égrène quelques notes sur le clavier, Cassie sent que remonte en elle tout le passé, notamment le souvenir du rituel des récitals de printemps organisés par Miss Eckhart où se produisaient tous ses élèves, dont la rebelle Virgie Rainey, celle qui avala un bouton dans « Shower of Gold».

19 La nouvelle, très proustienne dans sa manière de reconstruire le passé par bribes, alterne le point de vue naïf du jeune Loch, auquel les intentions incendiaires de Miss Eckhart échappent largement, avec celui, plus informé, de sa sœur. Loch, alité, a tout loisir d'épier les mouvements chaotiques de ceux qui investissent la maison voisine. Cette maison à présent divisée est littéralement «lue» par Loch depuis le poste d'observation de la fenêtre de sa chambre. A l'étage, Virgie y donne des rendez-vous 
galants; au rez-de-chaussée, Miss Eckhart s'affaire autour du piano ; plus tard se présente de nouveau sur le porch King MacLain qui, de retour dans la maison qui fut la sienne, tombe nez à nez avec la pyromane.

Loch, dont le nom évoque la serrure par homophonie, ou tout au moins une forme de verrouillage qu'il s'agirait de déjouer, est une figure du lecteur tentant, souvent avec retard, de relier des fragments narratifs, des éléments épars, entraperçus par les fenêtres de la maison MacLain, elles-mêmes en partie obstruées par des stores baissés ou des effets d'ombre, image de la résistance d'un sens global où prolifèrent les angles morts, où perdure un sentiment d'obstruction, tenace dans cette nouvelle comme dans tout le recueil. Comme pour souligner cette mise-en-abyme de l'acte de lecture, le pignon de la maison compte six fenêtres... plus une, autant que de nouvelles dans le cycle : " In the side of the house were six windows (...), and back of the chimney a small stair window shaped like a keyhole » $(1949,275-76)^{10}$.

21 Les erreurs de jugement de Loch, qui se méprend sur l'identité de Miss Eckhart et de King MacLain, sont aussi les nôtres, mais elles sont plus que cela : elles produisent une sorte d'épaississement fantasmagorique de la trame narrative, ouvrant des possibles que l'issue de la nouvelle ne confirmera pas, mais qui n'en ont pas moins d'existence dans l'esprit du lecteur comme dans celui de l'enfant candide. Ainsi, il n'est pas rare, dans cette nouvelle comme dans les autres, que le potentiel de sens déduit localement entre en contradiction avec le sens reconstruit globalement, l'imbrication du local dans le global ne se faisant pas ou se faisant mal. Ces sens possibles ne cessent pas, pour autant, de s'appuyer sur des liens virtuels entre telle occurrence et telle autre à quoi le cycle invite en permanence et auquel il fait place dans son programme, même si ces liens s'avèrent relever finalement d'une méprise ou d'une fausse piste.

Par ailleurs, le modèle cinématographique n'est sans doute pas extérieur à l'inspiration de Welty dans «June Recital ». Outre le fait que Virgie joue du piano sur les films muets présentés au Bijou, le cinéma de Morgana, et que le cinéma est souvent convoqué comme référence privilégiée dans le texte (McWhirter 73), il est clair que le récit se construit comme un montage de séquences proche de la grammaire cinématographique, l'œil étant invité à reconstruire une fluidité visuelle et narrative à partir d'une simple juxtaposition d'images aperçues comme au travers de trous de serrure. Plus largement, la pellicule en tant que support du langage cinématographique, avec ses vingt-quatre images par seconde créant une impression de continuité à partir d'une discontinuité, fournit au cycle de nouvelles un assez bon équivalent puisqu'elle est à la fois flux et ellipse, découpage et unité, inscrivant matériellement le manque dans son déroulement.

\section{« Sir Rabbit » ou le récit mythique en morceaux}

"Sir Rabbit », la courte nouvelle qui suit " June Recital », réintroduit le fantasque King MacLain au gré de ses pérégrinations. Cette fois, il chasse la caille dans les bois de Morgana, mais cela ne trompe personne : ce sont des rencontres féminines qu'il espère. Lorsque Mattie Will Sojourner, jeune femme de la campagne des alentours de Morgana, et épouse du rustique Junior Holifield, croise le chemin de King, ce qui advient prend la forme d'un viol aux forts échos mythologiques où sont convoquées, en filigrane, les figures de Léda et de Zeus, doubles illustres des personnages (après Danaé et Zeus dans "Shower of Gold ») $)^{11}$. Dans ce texte très profondément énigmatique lorsqu'il évoque le 
viol en termes voilés ou euphémisés, Mattie Will, fascinée par le légendaire séducteur de Morgana, devient à son corps défendant partie intégrante de la légende, au même titre que toutes celles qu'il a soumises :

she was Mr. MacLain's Doom, or Mr. MacLain's Weakness, like the rest, and neither Mrs. Junior Holifield nor Mattie Will Sojourner; now she was something she had always heard of. $(1949,338)$

no matter what happened to her, she had to remember, disappointments are not to be borne by Mr. MacLain, or he'll go away again. $(1949,338)$

La légende est un texte tyrannique qui requiert qu'on le déchiffre pour mieux s'y soumettre : c'est le texte de la domination masculine, dont Mattie Will perçoit cependant les failles lorsque King finit par s'endormir au pied d'un arbre après leurs ébats, ronflant, ridicule, désarticulé comme un pitoyable pantin :

she gazed at the sounding-off, sleeping head, and the neck like a little porch column in town, at the one hand, the other hand, the bent leg and the straight, all those parts looking no more driven than her man's now, or of any more use than a heap of cane thrown up by the mill and left in the pit to dry. (1949, 339-40)

Il n'y a plus de corps mâle triomphant, ni même en action ${ }^{12}$, mais les parties d'un corps éclaté et sans usage. Le mythe de la séduction masculine est exposé pour ce qu'il est: un assemblage artificiel que la jeune femme déconstruit après avoir rêvé d'en être le point de fixation. Ce qu'elle déconstruit, c'est aussi le récit attaché à la figure masculine récurrente depuis le début du recueil, King, ce roi de Morgana qui apparaît et disparaît à sa guise comme un dieu antique, et dont on pouvait penser jusqu'alors qu'il confèrerait à l'ensemble une certaine unité, un fil conducteur héroïque : il n'en est rien. "All those parts »: le corps démembré de ce qui aurait pu être un héros est encore à l'image de ce cycle composite tournant le dos à la cohésion qu'avait failli lui prêter le souffle d'un récit légendaire ici hors d'haleine. De même, il ne reste dans cette description qu'une pauvre petite colonne (" a little porch column ») évoquant, derrière l'image à peine voilée d'une ruine sexuelle, la ruine d'un autre grand mythe sudiste, celui de la plantation tel que la " plantation romance " a pu l'idéaliser ${ }^{13}$.

Avant cette rencontre avec King, la nouvelle s'ouvrait sur une première partie où Mattie Will, encore adolescente, rencontrant par hasard les jumeaux MacLain qui l'entraînaient dans des cabrioles au fond des bois, se méprenait sur leur identité et croyait d'abord avoir affaire à leur père en personne : « He looked around first one side of the tree and then the other. And not a word! » Puis elle prenait conscience de son erreur : «If it was Mr. King, he was, suddenly, looking around both sides of the tree at once-two eyes here and two eyes there, two little Adam's apples, and all those little brown hands " (1949, 331, mes italiques). Le texte nous soumet ici un contrat de lecture implicite à usage du cycle de nouvelles, lequel doit se lire à la fois dans la succession ( ( first one... and then the other ») et dans la simultanéité (« at once »). A la nouvelle en cours de lecture se superpose sans cesse le souvenir des précédentes et l'anticipation des suivantes, dans une sorte de feuilletage, comme dans l'esprit de Mattie Will se superposent la rencontre avec les jumeaux et celle avec leur père. D'une occurrence à l'autre, Mattie Will relit un texte qui est presque le même sans l'être tout à fait, puisque ses artifices finissent par éclater au grand jour et que le récit des hauts faits masculins est réduit en morceaux, ceux dont est fait le cycle composite. 


\section{« Moon Lake » ou l'interpénétration} entretient avec les autres textes les liens les plus tentaculaires, des fillettes de Morgana côtoient, le temps d'un été, des orphelines placées comme elles dans un camp de vacances au bord d'un lac voisin. L'une de ces orphelines, Easter, manque s'y noyer: c'est Loch Morrison, passé d'observateur dans «June Recital » à acteur ici, qui la sauve, mais la manière dont le sauvetage est décrit (Loch assis à califourchon sur le corps trempé de Easter), évoque autant le viol, écho de la nouvelle précédente, que la résurrection suggérée par son prénom. Les environs du lac, zone de contact entre classes sociales, entre sauvagerie et domestication, entre le familier et l'inexploré, entre innocence et expérience, spatialisent en quelque sorte certains enjeux de la lecture du cycle en ce que ce dernier nous amène lui aussi à faire se croiser, se mêler, se répondre, des espaces textuels différents d'une nouvelle à l'autre, forçant ainsi à l'hybridation comme le fait Moon Lake sur ses rives. Viennent aussi s'y reproduire ou s'inverser certains motifs diégétiques: les bois environnants sont le lieu où rôde en permanence la menace d'une présence masculine, celle des jumeaux MacLain ou de leur père, comme dans «Sir Rabbit »; munies d'un télescope, deux fillettes observent Loch Morrison qui se déshabille dans sa tente, situation inverse de celle de "June Recital ».

Au final, la cohabitation à laquelle Moon Lake contraint les fillettes de Morgana et les orphelines des faubourgs transfère dans le champ de la critique sociale le principe structurel du cycle qui force à coexister des éléments hétérogènes tout en en montrant les troublantes ressemblances. C'est ainsi que Nina Carmichael, petite fille de bonne famille, finit par reconnaître en Easter, l'orpheline rebelle, une mystérieuse part d'ellemême au potentiel dévastateur : "At least what had happened to Easter was out in the world, like the table itself. There it remained - mystery, if only for being hard and cruel and, by something Nina felt inside her body, murderous » (1949, 372, mes italiques). L'identification à l'autre, inscrite dans une expérience vécue par le corps lui-même (" inside her body ») abolit la distance sociale qui donnait le ton de la première partie de la nouvelle, et dont l'excipit souligne ici ironiquement l'inanité, l'essentiel étant que, comme dans l'expérience faite par le lecteur du cycle, ce qui passait pour autre peut être vu, en fait, comme un avatar du même.

\section{« The Whole World Knows » ou l'incomplétude}

Dans « The Whole World Knows », Ran, l'un des jumeaux MacLain, a épousé Jinny Love Stark, fille d'une des plus influentes familles de Morgana. Mais celle-ci vient de quitter le domicile conjugal, s'étant amourachée d'un autre. La nouvelle suit Ran dans ses errances alors que, par vengeance ou par dépit, il séduit Maideen, l'employée de la graineterie qui, bien vite abandonnée, finira par se suicider. Le titre de la nouvelle reprend les propos de Snowdie MacLain à son fils : "The whole world knows what she did to you » $(1949,390)$. En ne nommant pas ce qu'il y a à savoir, ce titre, initialement lu comme un énoncé elliptique autonome, est requalifié en aposiopèse lorsqu'il reparaît dans le dialogue, désignant ainsi un manque qui ouvre, pour le lecteur, une place à prendre, signe distinctif du cycle de nouvelles. Il est aussi, à l'évidence, le miroir inversé du titre d'une des nouvelles de Winesburg, Ohio sur le thème identique d'une 
séduction avortée : « Nobody Knows ». Au lecteur, donc, de restituer la part manquante en même temps que le double intertextuel.

Cette nouvelle soulève également des questions dont les réponses ne seront fournies que dans les deux nouvelles ultérieures. "How was I to know she would go and hurt herself? " $(1949,392)$ se demande Ran au sujet de Maideen. Or le suicide de Maideen ne sera explicitement évoqué que dans la dernière nouvelle du cycle. De même, Ran questionne aussi en pensée son père et son jumeau, tous deux absents : « Father, Eugene! What you went and found, was it better than this?» $(1949,392)$ Or, il faut attendre la nouvelle suivante pour apprendre que Eugene n'a guère trouvé le bonheur à San Francisco ; quant à King, de retour à Morgana pour de bon dans la dernière nouvelle, il ne dira pas si ses escapades amoureuses valaient mieux que la vie auprès de l'épouse qui lui est restée fidèle. Quoi qu'il en soit, « The Whole World Knows » se place sous les sombres auspices du manque : épouse enfuie, interlocuteurs absents, questions sans réponse, aventures insatisfaisantes. Cette incomplétude radicale, peut-être assez proche, au final, de ce qu'un jumeau peut éprouver en l'absence de son autre, se trouve, de fait, dédoublée dans le choix d'une forme narrative qui ne se suffit pas à elle-même, dépendante qu'elle est d'autres nouvelles pour trouver, ne serait-ce que temporairement, son sens. D'où la singulière ironie du titre, qui annonce un tout quand tout est manque, et qui affirme que l'on sait tout quand tant de faits font obstinément défaut.

31 Ces lacunes, le texte nous les donne presque à entendre dans la dernière scène de la nouvelle. Alors qu'il a emmené Maideen dans un motel, Ran est obsédé par le son d'un bâton qu'on traîne à dessein contre les barreaux d'une grille :

Then while she spoke to me I could hear all the noises of the place we were in - the frogs and night-birds of Sunset Oaks, and the little idiot nigger running up and down the fence, up and

down, as far as it went and back, sounding the palings with his stick. $(1949,392)$

Sorte d'équivalent sonore du modèle cinématographique de "June Recital », ce bruit produit une impression de continuité dans la discontinuité, un effet d'intermittence entre disparition et résurgence. Il nous ramène du même coup à notre expérience de lecture, et de relecture (" as far as it went and back»), qui est la reconstruction d'une séquence (narrative, musicale, filmique) sur la base d'espacements omniprésents.

\section{«Music from Spain » ou le corps étranger}

33 Unique nouvelle du cycle à n'être pas située à Morgana, "Music from Spain » fait clairement pendant à "The Whole World Knows " puisqu'on y découvre une journée dans la vie de l'autre jumeau, Eugene, marié et établi à San Francisco. Ce jour-là, après avoir giflé sa femme Emma sans raison apparente au petit-déjeuner, Eugene décide de ne pas aller travailler et s'engage dans une longue errance dans les rues de la ville, où il croise un guitariste espagnol entendu en concert la veille, et qu'il suit jusque sur une falaise au bord de l'océan, lieu d'une étrange étreinte teintée d'homoérotisme entre les deux hommes dangereusement près de basculer dans le vide. Le soir venu, Eugene finira pourtant par rentrer sagement chez lui.

34 A chaque page, la nouvelle pose implicitement la lancinante question de son appartenance au cycle, en même temps que celle de l'appartenance de Eugene à la communauté de Morgana qu'il a quittée, ainsi qu'à la norme hétérosexuelle que son comportement met un instant en péril. De nouveau, le texte propose un équivalent 
fictionnel du mode de fonctionnement du format cyclique: il s'agit, pour le dire trivialement, d'en être ou pas. Certes, l'escapade de Eugene rappelle fortement celles, répétées, de son père de légende, et celle de Ran avec Maideen; certes, le récit est émaillé de nombreux souvenirs de Morgana au fil de la longue déambulation du personnage à San Francisco. Mais le texte frappe surtout par la mention récurrente de présences étrangères dans la ville: en plus du musicien espagnol, qui ne parle pas l'anglais, Eugene croise Indiens, Chinois, Français, Polynésiens, Philippins. Or, la nouvelle tout entière apparaît à bien des égards comme un corps étranger dans le cycle, une sorte d'anomalie qui abandonne le Sud pour la côte ouest et la communauté rurale pour la mégalopole, produisant du même coup un effet de défamiliarisation.

Dès l'incipit, la voix narrative nous informe, au détour d'une phrase, que le couple a perdu une petite fille, morte de maladie infantile. Comme dans la nouvelle précédente, l'absence donne donc une inflexion décisive au récit de l'errance de Eugene. Welty semble suggérer qu'on ne saurait comprendre ce personnage si on ne cherche pas ailleurs que dans l'immédiateté de son cheminement. La ville qu'il parcourt est un texte à déchiffrer, complexe et tentaculaire, auquel seul un ailleurs peut donner sens : tout marcheur relie des points distincts et éloignés, abolissant une distance, rapprochant l'altérité d'un espace extérieur à son propre espace intérieur, processus similaire à la construction du sens pour le lecteur du cycle. Il nous faut, en effet, nous aussi, parcourir le présent du texte en restant sans cesse conscients d'un ailleurs qui fonde son incomplétude, mais qui, pour autant, n'explique pas tout. En alternant en permanence désorientation et reconnaissance, pour Eugene dans la ville comme pour le lecteur dans le texte, la nouvelle procède donc par cloisonnements et décloisonnements successifs, entre un présent apparemment peu cohérent et un passé censément éclairant, invitant ainsi le lecteur à ne pas s'en tenir à la linéarité du parcours de Eugene dans la ville pour privilégier une circulation fluide et signifiante, au-delà des frontières qui séparent l'ici de l'ailleurs, le même de son autre.

\section{«The Wanderers » : entre dénouement et renouement}

La dernière nouvelle du cycle, "The Wanderers ", resserre dans une certaine mesure les fils narratifs tissés depuis le début de l'ouvrage en convoquant tous les protagonistes, ou presque, à l'occasion de l'enterrement de Katie Rainey, narratrice de la première nouvelle. Ainsi se referme la boucle ouverte avec "Shower of Gold", englobant les autres textes du même coup et soumettant le lecteur à un puissant effet cyclique. C'est bien de fils narratifs qu'il est littéralement question ici puisqu'au moment où sa mère meurt sous ses yeux, Virgie Rainey, occupée par des travaux de couture comme les deux voisines dans "Shower of Gold", a des aiguilles entre les dents. Lorsque sa mère rend l'âme, Virgie entr'ouvre la bouche : " one by one the pins fell out on the floor" $(1949,431)$ - on se souvient du bouton régurgité dans la première nouvelle. La couture s'arrête donc lorsque la mort frappe la narratrice inaugurale et que le cycle touche à sa fin. Mais l'assemblage textile et textuel a la vie dure: les dernières pensées de la mourante vont à ses quilts, manifestations d'un art populaire très vivace dans le Sud qui offre comme un équivalent textile au format composite du cycle de nouvelles. 

plusieurs se fondent elles aussi sur un principe d'assemblage, comme l'évocation de cette vieille voiture qui transporte une famille venue rendre hommage à la défunte :

It was cracked like some put-together puzzle of the globe of the world. Its cracks didn't meet from one side across to the other, and it was all held together with straightened-out baling wire, for today. $(1949,439)$ les empêcher toutefois de flotter librement. Le lecteur attentif se souvient en effet des rubans de la capeline de Miss Snowdie dans «Shower of Gold» $(1949,265)$, des rubans de papier journal auxquels Miss Eckhart tentait de mettre le feu dans «June Recital » (1949, 283), et des "longs rubans de cette horrible matière verte" remontés des profondeurs du lac par Loch Morrison tentant de repêcher la noyée dans « Moon Lake » (1949, 363, ma traduction).

42 A propos de rubans, souvenons-nous encore du moment où Miss Eckhart, dans «June Recital », donnait ses instructions à ses jeunes élèves concernant la couleur des rubans qu'elles devraient nouer dans leurs cheveux le jour du récital :

Miss Eckhart decided early in the spring what color each child should wear, with what color sash and hair ribbon, and sent written word to the mother. She explained to the children that it was important which color followed which. 'Think of God's rainbow and its order' $(1949,309)$. 
chromatique : "You couldn't possibly have any idea what you would get when you untied your scarf» $(1949,286)$. Image d'une forme ouverte triomphante où domine l'incertitude factuelle et où chaque espace est appelé à déteindre sur l'espace voisin, l'écharpe ainsi réalisée modélise, là encore, le format cyclique.

\section{« Many dark ribbons »}

45 En guise de conclusion, revenons un instant sur les rubans de papier journal que s'apprête à brûler Miss Eckhart dans "June Recital» $(1949,282)$ : on sait que les journaux qu'elle utilise pour allumer l'incendie sont des exemplaires du Bugle, le quotidien de Morgana, c'est-à-dire une sorte de texte officiel ici déchiqueté, fragmenté. Brûler la maison, c'est d'ailleurs aussi reproduire un ingrédient diégétique récurrent de la "plantation romance", autre récit officiel s'il en est, ancré dans la veine romanesque. Pour autant, le geste de Miss Eckhart exprime assez bien l'ambiguïté du rapport au matériau textuel chez Welty. Il s'agit certes de démembrer les récits mythiques qui ont cours dans la communauté, récits ici découpés, amoindris, partiellement détruits, ou tournés en dérision, comme pour se libérer de leur poids. Mais dans le même temps, il s'agit aussi de les recoudre en une configuration neuve et méconnaissable. Le cycle dans son entier, dans son rapport aux grands mythes de la civilisation occidentale, incessamment présents en filigrane derrière l'anecdote sudiste, procède de même: il lacère et recompose, dénoue et renoue, méthode double et contradictoire inhérente à la forme du cycle, qui ne cesse elle-même d'exposer les manques de son tissu narratif déchiré et reprisé.

Face à ce matériau textuel révélant sa profonde duplicité, il n'est pas anodin que de nombreux protagonistes du cycle se trouvent placés dans une position de déchiffrement, mettant ainsi en abyme la position du lecteur. Dans « June Recital », par exemple, Miss Eckhart, assise au piano devant sa partition pendant un violent orage, joue avec passion un morceau de musique qui s'avérera ensuite ne pas être celui qu'elle semblait en train de lire : cet exemple met en lumière un irréductible écart entre ce qui est écrit et l'interprétation qui en est faite, la lecture du texte devenant ici une sorte de performance recréant autre chose que la lettre du texte.

De même, dans " Moon Lake ", Easter, l'orpheline qui manquera bientôt se noyer, trace dans le sable les lettres de son nom sous l'œil de deux fillettes de Morgana :

In clear, high-waisted letters the word 'Esther' cut into the sand. Then she jumped up.

'Who's that?' Nina asked.

Easter laid her thumb between her breasts, and walked about.

"Why, I call that 'Esther."

'Call it 'Esther' if you want to, I call it 'Easter.' $(1949,357)$

Ce dialogue met subtilement en scène la possibilité de « lire double » à laquelle le cycle de nouvelles nous met au défi : ce que le format cyclique interroge en nous, c'est notre aptitude à lire à chaque moment ce qui est écrit et autre chose que ce qui est écrit, à la fois le présent du texte et son fantôme démembré, dilué, fragmenté dans le reste du recueil, à la fois l'autonomie triomphante de "Easter » et son double "Esther " qui traînera toujours quelque part derrière elle, à l'image de chaque nouvelle et des rubans d'associations qui lui sont attachés et qu'elle charrie dans son sillage - «like suggestions and withdrawals of some bondage.» 


\section{Ouvrages cités}

ANDERSON, Sherwood. Winesburg, Ohio. 1919. New York et Londres : Norton Critical Edition, 1996.

ANDERSON, Sherwood. Sherwood Anderson's Memoirs. NewYork : Harcourt Brace, 1942.

ELIOT, T.S. "Ulysses, Order, and Myth." Selected Prose of T.S. Eliot. Ed. Kermode, Frank. San Diego: Harcourt, Farrar, Straus \& Giroux, 1975. 175-178.

INGRAM, Forrest L. Representative Short Story Cycles of the Twentieth Century. Studies in a Literary Genre. The Hague : Mouton, 1971.

JEWETT, Sara Orne. The Country of the Pointed Firs. 1896. New York : The Modern Library, 2000.

KELLEY, Margot Anne. "Gender and Genre: The Case of the Novel-in-Stories." American Women Short Story Writers : A Collection of Critical Essays. Ed. Brown, Julie. New York : Garland, 1995. 295-310.

KENNEDY, J. Gerald. Modern American Short Story Sequences. Cambridge : Cambridge University Press, 1995.

KREYLING, Michael. Author and Agent : Eudora Welty and Diarmuid Russell. New York : Farrar, Straus and Giroux, 1991.

LISTER, Rachel. « Female expansion and Masculine Immobilization in the Short Story Cycle. » Journal of the Short Story in English 48 (Spring 2007) : 1-12.

LUNDÉN, Rolf. The United Stories of America: Studies in the Short Story Composite. Amsterdam, Atlanta, GA: Rodopi, 1999.

LUSCHER, Robert. "The Short Story Sequence: An Open Book.” Short Story Theory at a Crossroads. Eds. Susan Lohafer and Jo Ellyn Clarey. Baton Rouge : Louisiana State University Press, 1989. 148-170.

MANN, Susan Garland. The Short Story Cycle. A Genre Companion and Reference Guide. Westport, CT.: Greemwood Press, 1989.

McWHIRTER, David. "Eudora Welty Goes to the Movies: Modernism, Regionalism, Global Media". Modern Fiction Studies, Vol. 55, №1 (Spring 2009) : 68-91.

SCHEVILL, James. Sherwood Anderson : His Life and Work. Denver : University of Denver Press, 1951.

VICTOR, Jean-Marc. "Présences du végétal dans The Golden Apples de Eudora Welty." Sillages critiques [En ligne], 22 | 2017, mis en ligne le 30 mars 2017, consulté le 08 juin 2021. URL : http:// journals.openedition.org/sillagescritiques/5119

WELTY, Eudora. A Curtain of Green. 1941. The Collected Stories of Eudora Welty. New York : Harcourt Brace Jovanovich, 1980. 1-149.

WELTY, Eudora. The Robber Bridegroom. 1942. New York : Harcourt Brace Jovanovich, 1970.

WELTY, Eudora. The Wide Net. 1943. The Collected Stories of Eudora Welty. New York : Harcourt Brace Jovanovich, 1980. 151-258.

WELTY, Eudora. Delta Wedding. 1946. Londres : Virago, Modern Classics, 1982. 
WELTY, Eudora. The Golden Apples. 1949. The Collected Stories of Eudora Welty. New York : Harcourt Brace Jovanovich, 1980. 259-461.

WELTY, Eudora. One Writer's Beginnings. 1984. New York : Warner, 1991.

WELTY, Eudora. The Eye of the Story: Selected Essays and Reviews. 1979. Londres : Virago, 1987.

WELTY, Eudora. Occasions. Selected Writings. Ed. Pearl Amelia McHaney. Jackson : University Press of Mississippi, 2009.

ZAGARELL, Sandra A. "Narrative of Community: The Identification of a Genre." Signs: Journal of Women in Culture and Society 13, no. 3 (1988) : 498-527.

\section{NOTES}

1. Sans doute ne doit-on pas s'étonner de voir se développer le cycle de nouvelles à l'époque moderniste, éprise de fragmentation, de discontinuité et d'éclatement formel (Kennedy xi).

2. Forrest Ingram est le premier à proposer une définition du cycle, qu'il place d'emblée sous le signe de la dualité : "Every story cycle displays a double tendency of asserting the individuality of its components on the one hand and of highlighting, on the other, the bonds of unity which make the many into a single whole » (19).

3. En 1949, date de publication de The Golden Apples, Welty a déjà à son actif deux recueils de nouvelles (A Curtain of Green, 1941, et The Wide Net, 1943), et une courte fantaisie, entre conte et récit historique, qualifiée de «novella (The Robber Bridegroom, 1942). Son seul roman en date (Delta Wedding, 1946), premier ouvrage à lui valoir un relatif succès éditorial, naît en réalité de la réécriture, lente et douloureuse, d'une nouvelle restée inaboutie. Comme le rappelle Michael Kreyling, "She had always justified her career as a short-story writer in large measure in the belief that the short story was a legitimate literary genre in its own right, and her genre by natural skill and inclination. [...] She had resisted all the opinions that the novel was intrinsically a greater achievement " (95).

4. Margot Kelley souligne d'ailleurs une nette sur-représentation des femmes dans le recours au format cyclique, lui-même indéniablement minoritaire (296).

5. On ne reviendra pas ici sur la controverse terminologique qui voit s'affronter divers vocables pour désigner au final le même objet littéraire hybride : "short story sequence », « composite novel », « short story composite », «story-cluster » (sur ce point, voir Lundén 12-18).

6. Notons qu'elle est tout aussi familière du cycle de Jewett (Welty 1979, 48-49).

7. Gerald Kennedy invite à ne pas négliger l'influence de considérations économiques sur le succès du format cyclique : nous parlons ici de textes qui peuvent "se vendre " deux fois, d'abord isolément dans des magazines, puis en groupe sous la forme d'un volume unique (Kennedy viii-ix).

8. Les métaphores de liens souterrains et d'affleurement utilisées ici invitent également à lire The Golden Apples selon des modalisations inspirées du monde végétal, qui ont fait l'objet d'une précédente étude (voir Victor).

9. L'ancrage décisif dans un territoire, trait commun aux cycles de Jewett, Anderson et Welty, est une tendance régionaliste généralement marquée dans ce genre narratif (Zagarell 499-500). 
10. En créant cette maison aux sept fenêtres, il n'est pas impossible que Welty, en adepte d'une mise en suspens de toute possibilité d'interprétation définitive, fasse obliquement allégeance à une tradition remontant à Hawthorne.

11. Il est clair que l'usage fait par Welty de tels échos mythologiques la place en droite ligne de l'héritage moderniste et de la méthode mythique de Joyce telle que la définit T.S. Eliot dans son article fondateur « Ulysses, Order, and Myth. »

12. A propos de King et des deux fils MacLain, Rachel Lister parle de "masculine immobilization » (6).

13. Les manques constitutifs du format du cycle de nouvelles évoquent, d'ailleurs, à plus d'un titre, une autre ruine encore : celle du modèle romanesque.

\section{RÉSUMÉS}

Face à la tyrannie éditoriale du roman dans le champ de la fiction américaine, un certain nombre d'auteurs de la première moitié du XX $\mathrm{XX}^{\mathrm{e}}$ siècle s'écartent des conventions formelles qui président aux modalités du récit en explorant les possibilités offertes par le «cycle de nouvelles »: dans le cadre de ce format narratif singulier, chaque nouvelle du cycle peut se lire de manière autonome, mais son sens se trouve modifié et/ou accru par les rapports qu'elle entretient avec les autres textes du volume. La lecture se fait sur un mode discontinu et fragmentaire, où seuls divers types d'échos peuvent inciter à reconstruire une unité qui n'est pas donnée au départ, et qui sollicite beaucoup plus fortement que le roman - la participation active du lecteur. Cet article se propose d'analyser la complexité des jeux de format induits par la lecture d'un cycle de nouvelles exemplaire à plusieurs titres: The Golden Apples de Eudora Welty (1949). Comment ce format cyclique instable interagit-il avec la ligne thématique du livre, tendue entre liaison et déliaison et, du même coup, en quoi peut-il mener à une réflexion plus large sur les modalités de réception de cet objet narratif en rupture avec les normes romanesques?

In reaction to the novel's editorial tyranny in American fiction, a number of writers in the first half of the $20^{\text {th }}$ century shifting away from conventional narrative forms explored the possibilities of the short story cycle : within this peculiar narrative format, each story can be read independently but its meaning is modified and/or expanded through its relations to the other texts composing the volume. Reading becomes discontinuous and fragmentary. Various types of echoes invite the reader to reconstruct some degree of unity as such unity is not provided from the start, which requires a much higher level of reader participation than in the case of a novel. This article proposes to analyze the complex ways in which Eudora Welty plays with such a format in her own 1949 short story cycle The Golden Apples. How does this unstable cyclical format interact with the book's thematics, oscillating between interrelatedness and separateness and, as a result, how does it challenge the reader's own reception of this narrative construct that turns its back on novelistic norms? 
INDEX

Mots-clés : format, cycle de nouvelles, Welty (Eudora), Golden Apples (The), Anderson

(Sherwood), Winesburg Ohio, réception, théorie de la lecture, interdépendance, fragmentation

Keywords : format, short story cycle, Welty (Eudora), Golden Apples (The), Anderson

(Sherwood), Winesburg Ohio, reception, reading theory, interrelatedness, fragmentation

\section{AUTEUR}

\section{JEAN-MARC VICTOR}

Sorbonne Université

Jean-Marc Victor, ancien élève de l'Ecole Normale Supérieure de Fontenay-Saint-Cloud, est maître de conférences à Sorbonne Université, où il enseigne la littérature, la traduction et l'analyse de l'image. Il a publié divers articles sur la littérature du Sud des Etats-Unis (Eudora Welty, Flannery O'Connor, William Faulkner), sur la nouvelle (Janet Frame, Alice Munro) ainsi que sur la photographie américaine (Ralph Eugene Meatyard, Nancy Rexroth). Il est également le traducteur d'un recueil de nouvelles de l'Australien Patrick White (paru en 2021 chez Gallimard, collection Du Monde entier, en collaboration avec Nathalie Pavec). 